\title{
Effect of Selected Yogic Practices on Vital Capacity among University Men Students
}

\author{
Dr. Baljit Singh Sekhon ${ }^{1}$, Dr. P. V. Shelvam ${ }^{2}$ \\ ${ }^{1}$ Deputy Director of Sports and HOD, Nagaland University, Lumami, Nagaland, India \\ ${ }^{2}$ Professor, Department of Physical Education and Sports Sciences, Annamalai University, Tamil Nadu, India
}

\begin{abstract}
The purpose of the study was to find out the effect of selected yogic practices on vital capacity among University men students. To achieve this purpose of the study, thirty men students were selected as subjects who were from the various faculties, Nagaland University, Lumami. The selected subjects were aged between 19 to 24 years. They were divided into two equal groups of fifteen each, Group I underwent yogic exercise practice and group II acted as control that did not participate in any special training apart from their regular sports and games practices. The subjects were tested on selected criterion variables such as vo max prior to any immediately after the training period. The selected criterion variable such as vo ${ }_{2}$ max was measuring by queen's college step test. The analysis of covariance (ANCOVA) was used to find out the significant differences if any, between the experimental group and control group on selected criterion variable. The 0.05 level of confidence was fixed to test the significance, which was considered as an appropriate. The result of the present study has revealed that there was a significant difference among the experimental and control group on $\boldsymbol{v o}_{2}$ max.
\end{abstract}

Keywords: Yoga Practice, Vital Capacity, Training, Hatha Yoga, Cardio-Respiratory Efficiency

\section{Introduction}

Yoga exercises and techniques derived from yoga indirectly affect technical and tactical preparation, yoga exercises and techniques also have significant, direct effects on the physical, psychological, theoretical preparation and on the regeneration of the strength process. Yoga asanas can be used for warm-up, cool-down, regeneration, compensation of muscle misbalances, synthesis of mind and body, activation or deactivation of the body and as supplemental exercise. Yogic techniques which aim at physical and mental self-culture, have convincing scientific bases and produce consistent physiological changes. It has been reported that yogis are capable of achieving remarkable feats of endurance and controlling their autonomic functions. There is evidence that the practice of yoga improves cardiorespiratory efficiency and performance quotient. There is a need to have yoga better recognized by the health care community as a complement to conventional medical care. Over the last 10 years, a growing number of research studies have shown the practice of Hatha Yoga can improve strength and flexibility and may help control such physiological variables as blood pressure, respiration and heart rate, and metabolic rate to improve overall exercise capacity. Exponents of yoga believes that other exercise systems only have a physical beneficial effect on the body whereas yogasanas result in the development of the physical, mental, spiritual well-being, physical exercise performed vigorously are helpful in develop in muscles and flexibility in health people. In view of the fact the heart, lungs and respiratory system have to work much harder in other forms of physical exercises, exponents of yoga believe that this is an unnecessary release of vital energy. According to them asanas are different from physical exercises, since they are performed slowly with relaxation and concentration. This results in the nervous system, endocrine system, muscular system and the internal organs being benefited. The term, exercise is often applied to asanas but asanas should never be confused with an exercise. The world exercise gives us an idea of quick and forceful movements of the body or its parts and repeated action which leads to exertion, tension and fatigue. Asanas, on the other hand are practiced slowly and steadily which bring about physical and mental relaxation.

\section{Methodology}

The purpose of the study was to find out the effect of selected yogic practice on vital capacity among University men students. To achieve the purpose of the study, thirty men students were selected as subjects who were from the various faculties, Nagaland University Lumami. The selected subjects were aged between 19 to 24 years. They were divided into two equal groups of fifteen each, Group I underwent yogic exercise practice and group II acted as control that did not participate in any special training apart from their regular sports and games practices. The experimental group underwent the training program for three days per week for eight weeks. Among the selected variables such as vital capacity was measuring by wet spirometer. The data were collected at prior and immediately after the training program for the selected variable. Analysis of analysis of covariance (ANCOVA) was applied for analyze the data. In all the cases, 0.05 levels were used to test this significance.

\section{Results}

Findings: The mean and standard deviation scores of pretest, posttest and adjusted posttest of vital capacity on yogic exercise and control group are given in table. , $F^{\text {ee }}$ ratio test computed in regards to the vital capacity on yogic exercise and control group in the pretest, posttest and adjusted post test are also presented in table. 


\section{International Journal of Science and Research (IJSR) \\ ISSN (Online): 2319-7064}

Index Copernicus Value (2013): 6.14 | Impact Factor (2015): 6.391

Table: Mean Standard Deviation and „F $F^{c e}$ Ration of Yogic Exercise Group and Control Group on Vital Capacity

\begin{tabular}{|c|c|c|c|c|c|c|}
\hline \multirow{2}{*}{ Variables } & & \multicolumn{2}{|c|}{ Yogic Exercise } & \multicolumn{2}{c|}{ Control } & 'F' \\
\cline { 3 - 7 } & & Mean & S D & Mean & S D & ratio \\
\hline \multirow{2}{*}{$\begin{array}{c}\text { Vital } \\
\text { Capacity }\end{array}$} & Pretest & 3.52 & 0.27 & 3.51 & 0.30 & 0.16 \\
\cline { 2 - 7 } & Posttest & 3.72 & 0.31 & 3.52 & 0.26 & $4.69^{*}$ \\
\cline { 2 - 6 } & Adjusted posttest & 3.69 & & 3.51 & & $7.62 *$ \\
\hline
\end{tabular}

Table shows the analyzed data of vital capacity. The vital capacity pre means were 3.52 for yogic exercise group and 3.51 for the control group. The resultant, $\mathrm{F}^{\mathrm{ee}}$ ratio of 0.16 was not significant at .05 levels indicating that the two groups were no significant variation. The post test means were 3.72 for the yogic exercise group and 3.52 for the control group. The resultant , $\mathrm{F}^{\mathrm{ee}}$ ration of 4.69 at .05 level indicating that it was significant. The difference between the adjusted post-test of 3.69 for the yogic exercise group and 3.51 for the control group yield on , $\mathrm{F}^{\text {ee }}$ ration 7.62 which was significant at .05 level.

\section{Discussion and Conclusions}

The findings of the study showed that there was no significant difference between the pretest of vital capacity.

The findings of the study showed that there was s a significant difference between the posttest and adjusted posttest of vital capacity.

The results of the study have shown there was a significant difference among yogic exercise group and control group on vital capacity reference to the past studies on vo 2 max in accordance with Doijad, Kamble and Surdi, Bowman and Bhutkar.

\section{References}

[1] Aladar Kogler, Yoga for Every Athlete, (Mumbai: Jaico Publishing House, 2003) 2.

[2] Madanmohan, et. Al., "Effect of Yoga Training on Reaction Time, Respiratory Endurance and Muslce Strength", Indian J. Physiol Pharmac., 36(4), (1992), 229.

[3] James A. Raub, "Psychologic Effects of Hatha Yoga on Musculoskeletal and Cardiopulmonary Function", The Journal of Alternative and Complementary Medicine, 2002; vol 8 No 6: 797-812.

[4] A.J. Kennedy, Fitness: A Way of Life (New Delhi: Tata McGraw Hill Publishing Company Ltd, 1988) 159.

[5] M.M. Gore, Anatomy and Physiology for Yogic Practices (Bombay: B.M. Gore Publishers, 1991)

[6] David H. Clarke and H.Harrison Clarke, Advanced Statistics, (New jersey: Prentice Hall Inc., 1988), 3138.

[7] Doijad Vanayak P., Prathamesh Kamble and Surdi Anil D. "Effect of Yogic exercises on aerobic capacity", International Journal of Recent Trends in Science and Technology, Volume 6, Issue 3, 2013 pp 119-121.

[8] Balasubramanian B and Pansare MS., "Effect of yoga on anaerobic power of muscles", Indian J Physical Pharmacol 1991 Oct; 35(4): 281-2.
[9] Bowman AJ, et al., "Effects of aerobic exercise training and yoga on the baroreflex in healthy elderly persons", Eur J Clin Invest. 1997 May; 27(5):443-9.

[10] Bhutkar Pratima M. et al., "Effect of Suruanamaskar Practice on Cardio-respiratory Fitness Parameters", Al Ameen J Med Sci (2008)1 (2):126-129. 